\title{
Publisher's Note: Extreme-Ultraviolet Vortices from a Free-Electron Laser [Phys. Rev. X 7, 031036 (2017)]
}

Primož Rebernik Ribič, Benedikt Rösner, David Gauthier, Enrico Allaria, Florian Döring, Laura Foglia, Luca Giannessi, Nicola Mahne, Michele Manfredda, Claudio Masciovecchio, Riccardo Mincigrucci, Najmeh Mirian, Emiliano Principi, Eléonore Roussel, Alberto Simoncig, Simone Spampinati, Christian David, and Giovanni De Ninno

(Q) (Received 18 July 2018; published 27 July 2018)

DOI: 10.1103/PhysRevX.8.039901

This paper was published online on 28 August 2017 with an omission of a support statement in the Acknowledgments on page 7. "We acknowledge funding support from the Slovenian Research Agency under Research Project No. J1-8134" has been added as of 19 July 2018. The Acknowledgments are incorrect in the printed version of the journal.

Published by the American Physical Society under the terms of the Creative Commons Attribution 4.0 International license. Further distribution of this work must maintain attribution to the author(s) and the published articles title, journal citation, and DOI. 